\title{
Vehicular Node Localization Using Received-Signal-Strength Indicator
}

\author{
Ryan Parker and Shahrokh Valaee, Senior Member, IEEE
}

\begin{abstract}
Vehicle-to-vehicle communications via dedicatedshort-range-communication (DSRC) devices will enable safety applications such as cooperative collision warning. These devices use the IEEE 802.11p standard to support low-latency vehicleto-vehicle and vehicle-to-infrastructure communications. However, a major challenge for the cooperative collision warning is to accurately determine the location of vehicles. In this paper, we present a novel cooperative-vehicle-position-estimation algorithm which can achieve a higher accuracy and more reliability than the existing global-positioning-system-based positioning solutions by making use of intervehicle-distance measurements taken by a radio-ranging technique. Our algorithm uses signal-strengthbased intervehicle-distance measurements, vehicle kinematics, and road maps to estimate the relative positions of vehicles in a cluster. We have analyzed our algorithm by examining its performance-bound, computational-complexity, and communicationoverhead requirements. In addition, we have shown that the accuracy of our algorithm is superior to previously proposed localization algorithms.
\end{abstract}

Index Terms-Dedicated short-range communication (DSRC), IEEE802.11p, localization, position estimation, vehicular networks, wireless access in vehicular environment, wireless communication.

\section{INTRODUCTION}

$\mathbf{T}$ HE RECENT introduction of dedicated-short-rangecommunication (DSRC) devices, based on the IEEE $802.11 \mathrm{p}$ standard for wireless access in vehicular environments, has evoked considerable interest within the research communities and automotive industries. DSRC is designed to support high-speed, short-range, low-latency wireless communications between vehicles and between vehicles and roadside wireless infrastructure. The $802.11 \mathrm{p}$ amendment is indeed an enhancement of 802.11a in which node association has significantly been simplified to enable rapid connection establishment and network acquisition.

A DSRC device is categorized as an onboard unit, if built inside a vehicle, or a roadside unit (RSU) if mounted on roadside posts. DSRC devices will support a variety of safety applications, such as collision avoidance and road-hazard warnings, and other intelligent-transportation-system applications

Manuscript received February 20, 2007; revised May 30, 2007 and July 14, 2007. This work was supported in part by an in-kind contribution from Mark IV Industries. The review of this paper was coordinated by Prof. X. Shen.

R. Parker is with Microsoft, Redmond, WA 98052 USA (e-mail: rparker@ comm.utoronto.ca).

S. Valaee is with the Department of Electrical and Computer Engineering, University of Toronto, Toronto, ON M5S 3G4, Canada (e-mail: valaee@comm. utoronto.ca).

Color versions of one or more of the figures in this paper are available online at http://ieeexplore.ieee.org.

Digital Object Identifier 10.1109/TVT.2007.907687 such as electronic toll collection and real-time traffic advisories [1]. Safety applications alone have a huge potential to save tens of thousands of lives and billions of dollars. In the U.S. alone, the National Highway Traffic Safety Administration reports that vehicle crashes account for more than 40000 deaths, 3 million injuries, and an estimated economic loss of 230 billion dollars annually [2].

One of the most promising vehicular safety applications is the development of an advanced cooperative-collision-warning system. It is envisioned that the advanced vehicle-collisionwarning system will use vehicle-to-vehicle radio communications to create a cooperative collision-warning system, where vehicles-equipped with DSRC devices-cooperatively share information (i.e., location, speed, heading, acceleration, etc.) for collision anticipation. By sharing this information between peers, each vehicle is able to predict potential hazards and to take proper steps to alleviate the problem. However, accurately determining a vehicle's relative location among its neighbors still remains a fundamental challenge. Without accurate vehicle position estimates, the system may alert the driver when there is no danger of collision, creating a number of unwanted warnings, or may fail to alert the driver when there is a potential danger ahead.

This paper will focus on the importance of accurate position information in providing reliable safety applications, such as cooperative collision avoidance. We will first highlight some of the scenarios where position information and vehicle-tovehicle communication can be used to improve safety. Next, we will examine some of the existing techniques for vehicle positioning and the problems of those techniques and propose a novel algorithm.

\section{A. Safety Applications}

There are numerous scenarios where intervehicle communication can be used to improve the safety of passengers. Consider a vehicle at the head of a platoon which encounters an emergency event and is forced to suddenly stop. Typically, drivers rely on the brake lights of the vehicle immediately ahead of them to decide on their own braking action. However, if the emergency event is triggered by several cars ahead, by the time the car immediately ahead brakes, it may be too late to safely stop in time. The time for the driver to process the brake light ahead and to step on the brake (typically $0.75-1.5 \mathrm{~s}$ ) compounds the problem, potentially leading to an emergency event causing a multivehicle accident.

Chain vehicle collisions can be prevented or reduced in severity if the delay between the time of the event and the 


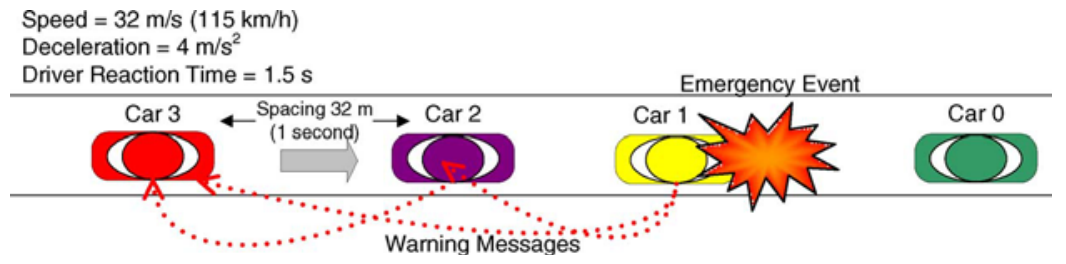

Fig. 1. Model for chain of car collisions.

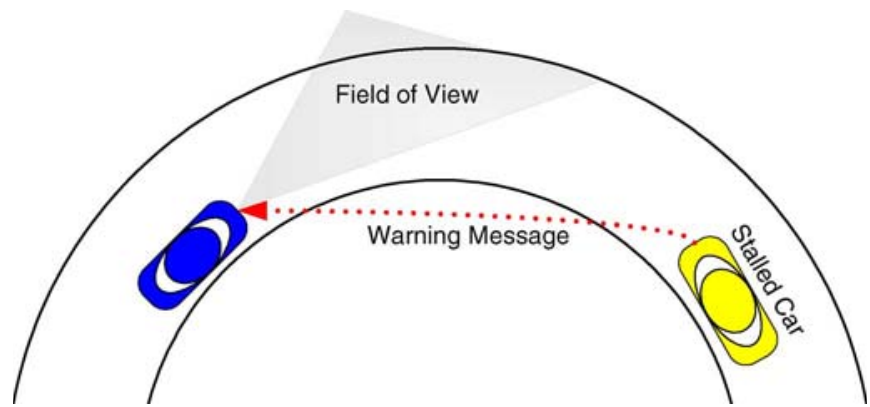

Fig. 2. Car is out of the driver's field of view, but using vehicle-to-vehicle communication, the driver can be forewarned.

time the trailing vehicles are informed of the event is reduced. Consider the scenario shown in Fig. 1, where all cars are traveling at $115 \mathrm{~km} / \mathrm{h}$ with an intervehicle spacing of $1 \mathrm{~s}$ and where car 1 encounters an emergency event and begins decelerating at $4 \mathrm{~m} / \mathrm{s}^{2}$. Car 2 starts decelerating after seeing the brake lights of car 1, and car 3 starts decelerating after seeing the brake lights of car 2. For this example, it was shown by Tatchitkou et al. [3] that without intervehicle communication, these cars will collide, and if the cars sent safety-warning messages containing position information, an accident would be prevented. They also showed that, in general, sending safety-warning messages with position information can substantially reduce the probability of collision within a platoon.

Safety-warning messages can also aid in expanding a driver's awareness of potential hazards. Consider the illustration in Fig. 2 where a car is stalled in the middle of the road around a blind corner. By the time the driver of the following car sees the vehicle and reacts, it may be too late to avoid collision. By sending a warning message containing position information to the oncoming vehicles, a driver can be forewarned of the potential hazards and can take appropriate action to avoid collision.

Both of these scenarios exemplify the importance of positioning information. In addition, these examples highlight the idea that when vehicles are equipped with DSRC devices, drivers gain an expanded awareness of their surrounding, giving them more time to react to potential road hazards.

In Section II, we review the related literature on radioranging techniques and wireless-node localization. We present our algorithm in Section III. In Section IV, we study the performance of our algorithm by deriving the Cramér-Rao bound (CRB) and the computational cost. In Section V, we present the simulation results and conclude the paper in Section VI.

\section{LOCATION-ESTIMATION TECHNIQUES}

From the aforementioned examples, it can be seen that the accurate estimation of the relative positions of neighboring vehicles is a vital component of vehicular-network safety applications. Designing a solution for the accurate estimation of neighboring vehicles based on real-time exchange of position estimates, using vehicle-to-vehicle communication, is a challenging task. Given the subsecond decision latency requirement of cooperative-collision-warning systems, the solution must be able to establish the relative position of all neighbors in real time and to continuously track their motion to proactively identify potential vehicle-collision scenarios.

Currently, global positioning system (GPS) is used to localize vehicles. The GPS unit locates itself by comparing the signal received from four or more GPS satellites. Although the GPS is fairly accurate in flat open areas where a line of sight to multiple satellites is possible, it fails to operate in tunnels and in downtown areas where blockage of satellite signals is frequent. Typically, during short outages of the GPS, vehicles will use a dead-reckoning system to maintain an estimate of their position [4]. Currently, the best dead-reckoning systems have horizontal accuracies of approximately $1 \%$ of the total distance traveled. Therefore, when traveling at $100 \mathrm{~km} / \mathrm{h}$, the dead-reckoning system can estimate the vehicle's location (with permissible error of $<10 \mathrm{~m}$ ) for $30 \mathrm{~s}$.

One of the main challenges identified by Miller and Huang [5] is that accurate location estimates were critical to the success of cooperative-collision-warning systems and that the approximate 5-m accuracy of a regular GPS is inadequate for such applications. Improved solutions, such as the differential GPS [6] and the assisted GPS [7], have been proposed. However, the reliability of these location estimators still does not satisfy the needs of safety applications in vehicular networks, which require that the relative position of vehicles be accurately estimated. It has recently been argued that a combined solution using the GPS - or one of its improved variants - and the use of radio-based-ranging techniques to determine distance estimates between vehicles in a cluster can be used to increase the reliability of the location estimator [4].

The techniques proposed in this paper can be used with or without the GPS. If the GPS estimate is available, the proposed techniques in this paper can be used to increase the accuracy of the location estimates. In the absence of the GPS, the techniques are used to locate vehicles.

\section{A. Radio-Based-Ranging Techniques}

Radio-based-measurement technologies leverage signalpropagation characteristics to derive an estimate of the 
distance between the transmitter and the receiver. There are three common radio-ranging techniques used for nodal position estimation in ad hoc networks, namely, received-signal-strength indicator (RSSI), time of flight [e.g., time of arrival (ToA) or time difference of arrival], and angle of arrival (AoA). We will review each of these techniques and determine their feasibility for use in vehicular networks.

The RSSI technique is the most widely known and provides a low-cost means to estimate distance since it does not require any specialized hardware [4], [8]. This technique uses known mathematical wireless-channel models that describe the relationships between path-loss signal attenuation and distance between the transmitter and receiver. One of the issues with this technique is that it does not always produce accurate distance measurements since there is large variation in signal attenuation in different environments, particularly when multipath and shadowing effects are present [9].

The time-of-flight-based methods find the distance by measuring the propagation delay of a signal from the transmitter to the receiver. In general, this technique works well with high-resolution time measurements, accurate real-time clock synchronization between nodes, and line-of-sight-propagation conditions. To keep an accurate real-time clock synchronization among all vehicles in a cluster would be difficult. However, McCrady et al. [10] presented a two-way reciprocal ToAranging technique that removes the need for any clock synchronization among receiver-transmitter pairs and provides high ranging accuracy $(<1 \mathrm{~m})$ even in multipath scenarios. The main drawback of this technique is the need for a complex hardware.

The AoA technologies estimate position coordinates of the subject node (e.g., vehicle) by using an antenna array and by measuring the AoAs of signals from neighboring nodes and then by performing triangulation. At close range, where line of sight is possible, this technique works well. However, as the distance between nodes increases, the performance degrades, particularly in scenarios where line of sight is not possible (e.g., urban environments), since the antenna array may lock onto a multipath component which would corrupt the angle measurements and introduce significant positioning errors.

The following two technologies could be applied to vehicular communications: the RSSI and the ToA systems. However, the key point to note is that, regardless of the radio-rangeestimation technology used, the distance measurements are inherently noisy as a result of a number of factors, including the limitations of the measurement device, multipath fading, shadowing, and nonline-of-sight errors. In addition, mobility complicates the case since outliers and noisy measurements can be misinterpreted as an observed motion and the effects of fading become more prevalent. Therefore, accurate localization of vehicles cannot solely depend on intervehicle-distance measurements using one of the aforementioned techniques.

\section{B. Localization of Wireless Nodes}

We will now examine some of the existing localization approaches. In [11], a GPS-free positioning algorithm has been proposed in which each node establishes a local coordinate

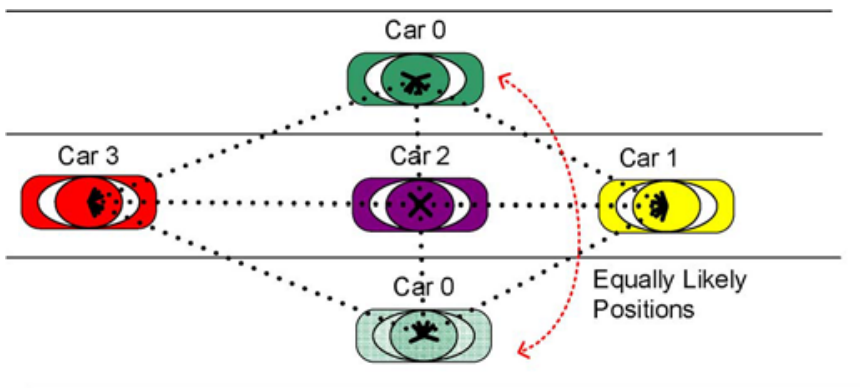

Fig. 3. Ambiguity in vehicle localization.

system based on the distance measurements between the nodes. Once the local coordinate systems are formed, the nodes align their coordinate systems to one of the nodes so that all coordinates are oriented in the same direction. Unfortunately, this algorithm requires that a large number of messages be exchanged between nodes. As a result, this procedure does not scale well and is not well suited for high-mobility environments, where nodes are frequently entering and exiting clusters. Iyengar and Sikdar [12] derived an improved version of Capkun et al. [11], to tackle these issues, by creating an algorithm which improves scalability and convergence times.

Kukshya et al. [4] used the results of Iyengar and Sikdar [12] as the bases for creating a method of localizing neighboring vehicles based on radio-range measurements. Their system consisted of the following three main components: 1) a clustering technique, which establishes a group of neighboring vehicles that exchange information and assigns master/slave associations; 2) a radio-based-ranging technique, which determines intervehicle-distance measurements; and 3) a positioning algorithm, which determines the relative position coordinates of the vehicles based on the distance measurements and triangulation. There are a few problems with this work. First, establishing a master/slave relationships may result in a large number of polling rounds to determine new masters for the cluster because the topology of vehicular networks is dynamic (i.e., vehicles are often entering and exiting different clusters). Second, the position estimates may become very inaccurate since the distance measurements are noisy. Therefore, each calculated relative coordinate system will contain some error in the position estimates, and, as the coordinate systems are aligned, the positioning error will propagate. Third, based on the distance measurements and triangulation alone, it is possible for a vehicle to have two equally likely positions. Consider Fig. 3, where car 0 has two possible positions that satisfy the same set of distance constraints.

This flaw in pure triangulation approaches (i.e., where it is possible for a node to have two or more positions that satisfy the same distance constraints) prompted the work by Eren et al. [13]. Their paper uses graph rigidity theory to derive the theoretical framework for determining which nodes have a unique position based on the internode-distance constraints.

Moore et al. [14] derived a robust-quads algorithm by modeling the network in a graph representation, with the vertices being the Euclidean positions of the nodes and the edges being the distances measured between the nodes. However, there are two types of discontinuous deformations that can prevent the 

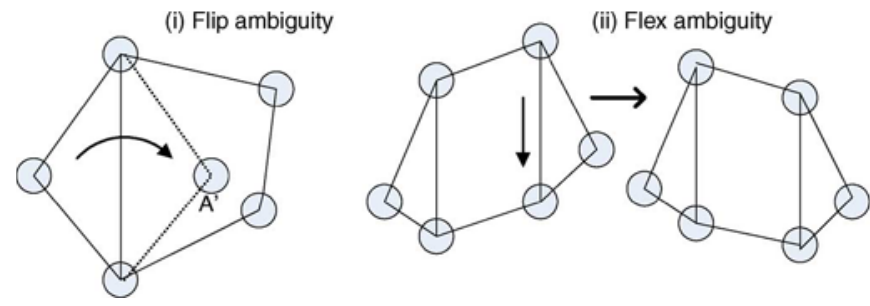

Fig. 4. (i) Flip ambiguity node A can be either A or A', and the distance constraints are still satisfied. (ii) Flex ambiguity where distance constraints are the same except that the graph is sheared.

graph realization from being unique (see Fig. 4): 1) a flip ambiguity where all the distance measurements are the same but the parts of the graph can become mirror reflections, or 2) flex ambiguities where the distance constraints remain the same but the graph is sheared. Moore et al. [14] proposed a heuristic to detect the nodes which have a high probability of having a unique realization. Their algorithm localizes only those nodes with unique realization to minimize the propagation of localization error when the coordinate systems are aligned to a common orientation.

The robust-quads algorithm [14] can be used in vehicular networks. The algorithm was used in a sensor network and had one experiment where a single node had mobility and the rest of the nodes remained stationary, showing that a mobile node could accurately be tracked within the network. However, it is still unclear how their algorithm would perform if all the nodes were mobile.

The majority of the existing algorithms have been targeted for use in wireless sensor networks. However, if instead we consider these algorithms for vehicular networks, some potential for further optimization arises. For example, with the knowledge that vehicles are constrained to travel, in general, within lanes, it may be possible to improve the accuracy and the complexity of the existing node localization algorithms since the search space for possible vehicle positions is smaller. Moreover, vehicles have knowledge of their kinematics (e.g., speed, heading, and acceleration); thus, the number of times the distance-measurement exchange takes place can be reduced. In addition, if the GPS is available, it can serve as an initial estimate for a vehicle's position. Then, through refinement, using the measured distances between vehicles, a more accurate position estimate may be found. It is speculated that an accurate and reliable solution for determining the relative positions of neighboring vehicles will not be solely based on one component (e.g., intervehicle-distance measurement) but on the result of a data fusion from a variety of information sources.

\section{Proposed Algorithm}

The proposed vehicle localization system is shown in Fig. 5, which is an extension of our earlier work in [15] to include road and lane boundaries and the associated mathematical analysis of these constraints. Furthermore, we have added a more rigorous analysis of the performance bounds by deriving the CRB in Section IV-A.

The localization module accepts inputs from the following four modules: 1) a ranging module, based on a radio-ranging technique, to estimate intervehicle-distance measurements; 2) an optional GPS module (if available) to provide an initial position estimate of the vehicle when there is high confidence in the GPS estimate (i.e., four or more satellites are in line of sight; 3) a vehicle sensor module to determine the vehicle's kinematics; and 4) a mapping module to ensure that the position estimates are within the road constraints. Using these four modules as inputs to the localization module, the following three-phase localization algorithm can be created.

\section{Phase 1: Initialization}

Obtain coarse initial position estimates for vehicles by measuring and sharing intervehicle distances to perform trilateration or by using a GPS.

\section{Phase 2: Refinement}

Improve the position estimates, by minimizing the error in the intervehicle-distance measurements, while ensuring that the road and lane constraints are met.

If the previous position estimates are available, ensure that the new position estimate is consistent with the vehicle-velocity constraints.

\section{Phase 3: Iteration}

Periodically iterate through phase 2 , using the final position estimate as the initial position estimate for subsequent intervals.

Consider a cluster of $n$ vehicles at unknown distinct locations in some physical region at time $t$. For each vehicle, we establish a map of the relative position of its neighbors. More explicitly, we estimate the true relative positions of the vehicles $\boldsymbol{A}$ defined as

$$
\boldsymbol{A}=\left[x_{1}, x_{2}, \ldots, x_{n}, y_{1}, y_{2}, \ldots, y_{n}\right]
$$

where $\left\{x_{i}, y_{i}\right\}_{i=1}^{n}$ represents the relative position of the $i$ th vehicle in a coordinate system with the location of the vehicle performing the mapping set at the origin $(0,0)$.

These position estimates will be created based on the following three main factors: 1) intervehicle-distance estimates, which are made by using a radio-based-ranging technology; 2) velocity information from each vehicle, which is derived from onboard sensors; and 3) using a road map to ensure that the position estimates are within the road boundaries. The first two factors were used in an earlier version of this paper [15], and the last factor will be included here. The road maps can be loaded via the RSU when the vehicle enters the corresponding fragment of the road.

A two-step process is performed to gain information about the intervehicle distances and velocities of all vehicles in the cluster. In the first step, intervehicle-distance measurements are made by each vehicle using a radio-ranging technique to estimate the vehicles' relative distance. In addition, in the first step, the vehicles within the cluster will read their own speed information. In the second step, the information each vehicle collected is shared with its neighbors, which can be accomplished by standard multicast techniques. Therefore, after the 


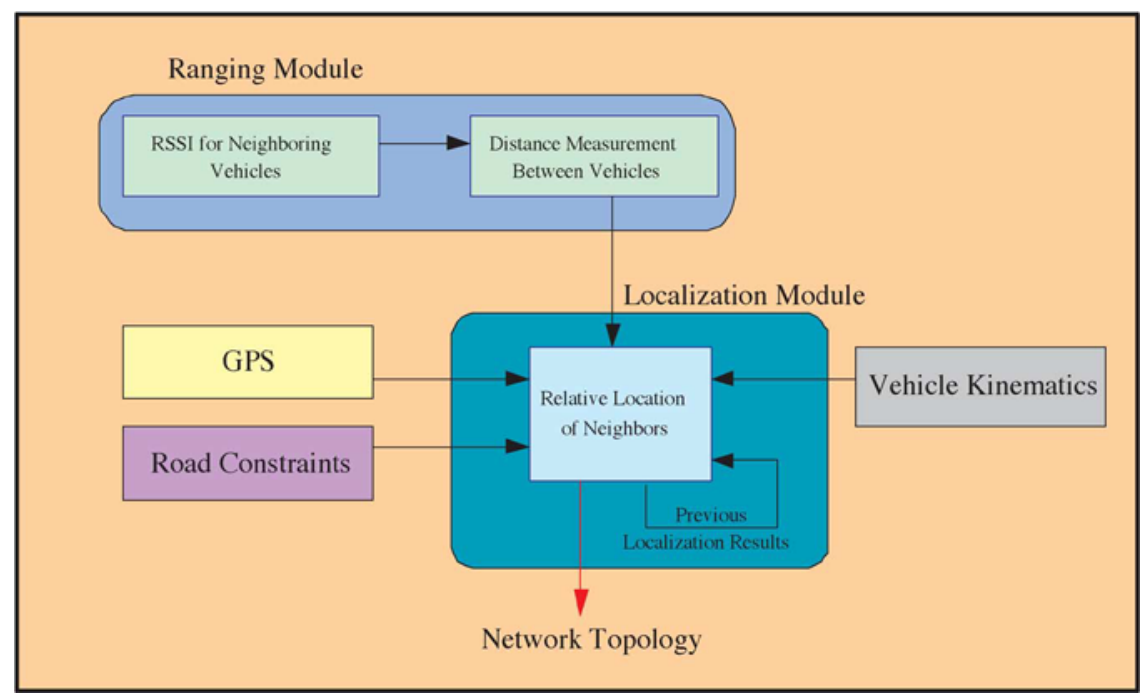

Fig. 5. Subsystem representation of the proposed solution.

second step, each vehicle has up to $n \times(n-1)$ intervehicledistance readings and a set of $n$ velocity readings. Note that the distance measured from vehicle $i$ to vehicle $j$ may not be equal to the distance measured from $j$ to $i$.

We use Kalman filtering since it is recursive, incorporates a motion model, and provides the optimal set of position estimates in the mean-square sense for Gaussian-noise distribution for the mobility and measurement models [16]. However, unlike [15], we will use here the road map to constrain the solutions to road boundaries. The motion model that we will use to incorporate the velocity measurements is

$$
\boldsymbol{A}_{k}=\boldsymbol{A}_{k-1}+T_{\mathrm{s}} \mathbf{u}_{k-1}+T_{\mathrm{s}} \mathbf{w}_{k-1}
$$

where

$$
\begin{aligned}
\boldsymbol{A}_{k} & =\left[x_{1, k}, \ldots, x_{n, k}, y_{1, k}, \ldots, y_{n, k}\right]^{\mathrm{T}} \\
\mathbf{u}_{k-1} & =\left[v_{x 1, k-1}, \ldots, v_{x n, k-1}, v_{y 1, k-1}, \ldots, v_{y n, k-1}\right]^{\mathrm{T}}
\end{aligned}
$$

where $n$ is the number of vehicles in the cluster at the $k$ th snapshot, $T_{\mathrm{S}}$ is the sampling interval, superscript $\mathrm{T}$ denotes transposition, $v_{x i, k-1}$ and $v_{y i, k-1}$ are the velocities of vehicle $i$ in the $x$ - and $y$-directions at time $k-1$, respectively, $\boldsymbol{A}_{k}$ is the position of vehicle at time $k$, and $\mathbf{w}_{k-1}$ is the process noise describing the mobility variations. We assume that $\mathbf{w}_{k-1}$ is a zero-mean Gaussian random variable, with the following covariance:

$$
\mathbf{Q}_{k-1} \triangleq \mathrm{E}\left\{\mathbf{w}_{k-1} \mathbf{w}_{k-1}^{\mathrm{T}}\right\}=\operatorname{diag}\left(\sigma_{x 1}^{2}, \ldots, \sigma_{x n}^{2}, \sigma_{y 1}^{2}, \ldots, \sigma_{y n}^{2}\right)
$$

where $\operatorname{diag}(\cdot)$ denotes a diagonal matrix.

Considering that vehicles usually move along the lanes in roads, the uncertainty in the direction of the road is greater than the uncertainty in the direction orthogonal to the road. Let us define the variance in the direction of the road for vehicle $i$ to be $\sigma_{i, a}^{2}$ and the variance in the direction orthogonal to be $\sigma_{i, o}^{2}$. Due to the higher uncertainty of the motion along the road, $\sigma_{i, a}^{2} \gg \sigma_{i, o}^{2}$. Thus, for a road that runs in a direction that is $\theta$ radians from the $y$-axis, the following transformation must be applied:

$$
\begin{aligned}
\sigma_{x i}^{2} & =\sigma_{i, o}^{2} \cos ^{2} \theta+\sigma_{i, a}^{2} \sin ^{2} \theta \\
\sigma_{y i}^{2} & =\sigma_{i, a}^{2} \cos ^{2} \theta+\sigma_{i, o}^{2} \sin ^{2} \theta .
\end{aligned}
$$

This allows $\mathbf{Q}_{k-1}$ to be biased in the direction of the road.

The observations of the intervehicle measurements are expressed as

$$
\mathbf{z}_{k}=\mathbf{h}_{k}(\boldsymbol{A})+\mathbf{v}_{k}
$$

where $\mathbf{h}_{k}(\boldsymbol{A})$ is a nonlinear equation describing the measurements at time $k$, and $\mathbf{v}_{k}$ is a zero-mean Gaussian random vector, with the covariance matrix $\mathbf{R}_{k}$, describing the noise characteristics of the measurements. The measurement equation is nonlinear since the distance between vehicles $i$ and $j$ equals $\sqrt{\left(x_{i}-x_{j}\right)^{2}+\left(y_{i}-y_{j}\right)^{2}}$. Note that the general form of the Kalman filter requires that the measurement (observation) equation be in a linear form; therefore, we will linearize (7) using the first-order Taylor-series expansion around the current position estimates. Let us define

$$
\hat{\mathbf{H}}_{k}=\left.\frac{d \mathbf{h}_{k}(\boldsymbol{A})}{d \boldsymbol{A}}\right|_{\boldsymbol{A}=\boldsymbol{A}_{k \mid k-1}}
$$

where $\hat{\mathbf{H}}_{k}$ can be referred to as the Jacobian matrix, and $\boldsymbol{A}_{k \mid k-1}$ is the estimate of $\boldsymbol{A}_{k}$ using the observation up to time $k-1$. Given (2) and the nonlinear form of (7), we can form the extended-Kalman-filter (EKF) algorithm. The size of the $\hat{\mathbf{H}}_{k}$ for a cluster of $n$ vehicles is $\left(n^{2}-n\right) \times(n-1)$. To reduce the computational complexity, we can reduce the number of rows in the $\hat{\mathbf{H}}_{k}$ matrix to $\left(n^{2}-n\right) / 2$ if we set the average of the pairwise distance measurements between vehicles $i$ and $j$.

The EKF algorithm can be viewed as the following set of recursive relationships:

$$
\begin{aligned}
\boldsymbol{A}_{k \mid k-1} & =\boldsymbol{A}_{k-1 \mid k-1}+T_{\mathrm{s}} \mathbf{u}_{k-1} \\
\mathbf{P}_{k \mid k-1} & =\mathbf{Q}_{k-1}+T_{\mathrm{s}}^{2} \Gamma_{k-1}+\mathbf{P}_{k-1 \mid k-1}
\end{aligned}
$$




$$
\begin{aligned}
\boldsymbol{A}_{k \mid k} & =\boldsymbol{A}_{k \mid k-1}+\mathbf{K}_{k}\left(\mathbf{z}_{k}-\mathbf{h}_{k}\left(\boldsymbol{A}_{k \mid k-1}\right)\right) \\
\mathbf{P}_{k \mid k} & =\mathbf{P}_{k \mid k-1}-\mathbf{K}_{k} \hat{\mathbf{H}}_{k} \mathbf{P}_{k \mid k-1}
\end{aligned}
$$

where $\Gamma_{k-1}$ is the covariance matrix describing the uncertainty in the velocity measurements, and $\mathbf{K}_{k}$ is the Kalman-filter gain defined as

$$
\mathbf{K}_{k}=\mathbf{P}_{k \mid k-1} \hat{\mathbf{H}}_{k}^{\mathrm{T}}\left(\hat{\mathbf{H}}_{k} \mathbf{P}_{k \mid k-1} \hat{\mathbf{H}}_{k}^{\mathrm{T}}+\mathbf{R}_{k}\right)^{-1} .
$$

The Kalman filter is initialized as

$$
\begin{aligned}
& \boldsymbol{A}_{0 \mid 0}=\mathbf{A}_{\mathrm{GPS}} \\
& \mathbf{P}_{0 \mid 0}=E\left[\left(\boldsymbol{A}_{0}-\mathbf{A}_{\mathrm{GPS}}\right)\left(\boldsymbol{A}_{0}-\mathbf{A}_{\mathrm{GPS}}\right)^{\mathrm{T}}\right]
\end{aligned}
$$

where $\mathbf{A}_{\mathrm{GPS}}$ is the vector of estimates from the GPS ${ }^{1}$, and $\mathbf{P}_{0 \mid 0}$ is the covariance matrix of the GPS estimates.

Once the location of the vehicles is estimated from the Kalman filter, the estimates should be checked against the road boundaries. Examples of road constraints can be suggested by enforcing road geometry. For example, each vehicle on the road should have an $x_{i}(t)$ position that satisfies

$$
L_{i} \leq x_{i}(t) \leq R_{i}
$$

where $L_{i}$ and $R_{i}$ are found from road width; similar bounds can be imposed on $y_{i}(t)$. These constraints can be represented as $D \boldsymbol{A} \leq d$, where $D$ is the selection matrix with the entities equal to $1,-1$, or 0 , and $d$ is the road boundaries. To enforce constraints, we project Kalman-filter estimates onto the constraint space [17] by solving

$$
\begin{gathered}
\tilde{A}_{k}=\arg \max _{\tilde{A}}\left(\tilde{A}-\boldsymbol{A}_{k \mid k}\right)^{\mathrm{T}} W\left(\tilde{A}-\boldsymbol{A}_{k \mid k}\right) \\
\text { such that } D \tilde{A} \leq d
\end{gathered}
$$

where $W$ is a positive-definite matrix chosen to give different weights to the elements of $\left(\tilde{A}-\boldsymbol{A}_{k \mid k}\right)$. This optimization is in the form of a quadratic programming problem [18] and is usually solved using an active set method.

Active set methods use the fact that only a small set of constraints is active at the solution. The active constraints in our problem are the ones for which the Kalman filter gives a coordinate that is outside the road boundaries. Assume that the active constraints are represented by $\tilde{D} \tilde{A} \leq \tilde{d}$ and then, replacing these constraints in (18), give the following solution:

$$
\tilde{A}_{k}=\boldsymbol{A}_{k \mid k}-W^{-1} \tilde{D}^{\mathrm{T}}\left(\tilde{D} W^{-1} \tilde{D}^{\mathrm{T}}\right)^{-1}\left(\tilde{D} \boldsymbol{A}_{k \mid k}-\tilde{d}\right) .
$$

This solution has a number of interesting properties [19]. First, the constrained estimate is unbiased. Second, setting $W=\mathbf{P}_{k}^{-1}$ results in a minimum variance filter. Third, setting $W=\mathbf{I}$ (where $\mathbf{I}$ is the identity matrix) results in a constrained estimate that is always closer to the true state than the unconstrained estimate.

Given the aforementioned set of equations for the Kalman filter, our algorithm can be summarized as follows:

Step 1) Each vehicle performs intervehicle-distance measurements and takes a reading of its own velocity.

${ }^{1}$ Instead of GPS, we may use triangulation to find the initial values.
This information is then shared with all the vehicles within the cluster. If new vehicles have just joined the cluster, an initial estimate of their position is also required. This initial estimate can be established in one of two ways, either by vehicles exchanging GPS position estimates or by measuring and exchanging the intervehicle distances to perform trilateration. In general, GPS, if available, is the preferred method in establishing initial estimates since trilateration, with noisy intervehicle-distance measurements, can often yield ambiguous initial position estimates [14].

Step 2) Update the (9) and (10) predictions. Note that the rank of the state matrix will be dynamic since the number of vehicles within the cluster can change from one time step to the next.

Step 3) Incorporate the measurements from step 1) to update (11) and (12). The current position estimate based on vehicle movements and a current set of intervehicledistance estimates will be contained in the matrix of position estimates $\boldsymbol{A}_{k \mid k}$ with an associated level of uncertainty captured by the $\mathbf{P}_{k \mid k}$ matrix. The algorithm also forces the position estimates to be within the road boundaries.

Step 4) Repeat steps 1), 2), and 3) at the update rate of the filter $T_{\mathrm{s}}$.

\section{Performance Analysis}

\section{A. Cramér-Rao Bound}

To gain a better understanding of the performance of our algorithm, we derive the CRB. The CRB provides a lower bound on the error covariance matrix of any unbiased estimate of unknown parameters. It is a tight bound in the sense that the maximum-likelihood detector asymptotically approaches the performance CRB for high signal-to-noise ratio [16]. For the case of localization, where velocity information is available, and using the motion model (2), the lower estimation bound denoted as $\boldsymbol{P}_{k}$ is calculated by the recursion [20]

$$
\begin{aligned}
\operatorname{Cov}\left(\boldsymbol{A}_{k}\right) & \geq \boldsymbol{P}_{k} \\
\boldsymbol{P}_{k+1} & =\left(\left(\boldsymbol{P}_{k}+T_{\mathrm{s}} \mathbf{Q}_{k}\right)^{-1}+\mathbf{J}_{\mathbf{P}}\right)^{-1}
\end{aligned}
$$

where $\mathbf{J}_{\mathbf{P}}$ is equal to the Fisher information matrix for the static localization case, which is studied for the received-signalstrength-, ToA-, and AoA-ranging techniques in [21]. The recursive (21) has the form of a Riccati recursive equation and will have a stationary form [20]

$$
\overline{\boldsymbol{P}}=\left(\left(\overline{\boldsymbol{P}}+T_{\mathrm{s}} \mathbf{Q}\right)^{-1}+\mathbf{J}_{\mathbf{P}}\right)^{-1}
$$

which has the following solution:

$$
\begin{aligned}
\overline{\boldsymbol{P}}=-\frac{1}{2} T_{\mathrm{s}} \mathbf{Q} & +\mathbf{J}_{\mathbf{P}}^{-\frac{1}{2}} \\
& \times\left(\mathbf{J}_{\mathbf{P}}^{\frac{1}{2}}\left(T_{\mathrm{s}} \mathbf{Q}+\frac{T_{\mathrm{s}}^{2}}{4} \mathbf{Q} \mathbf{J}_{\mathbf{P}} \mathbf{Q}\right) \mathbf{J}_{\mathbf{P}}^{\frac{1}{2}}\right)^{\frac{1}{2}} \mathbf{J}_{\mathbf{P}}^{-\frac{1}{2}} .
\end{aligned}
$$




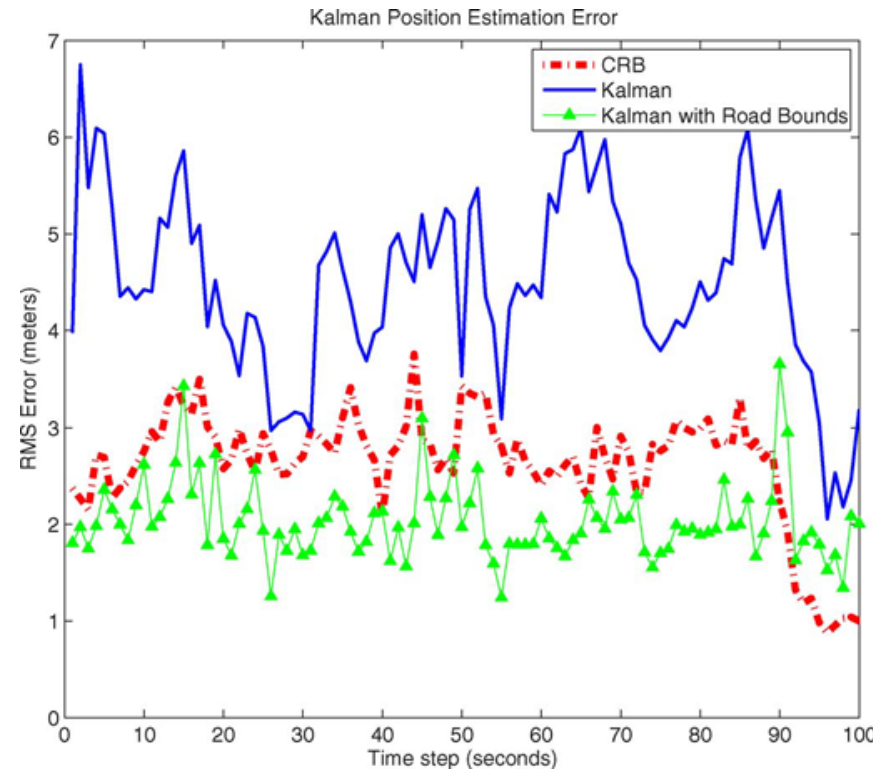

Fig. 6. CRB for position estimates versus our Kalman-filter-based solution; lower curve shows performance gain by forcing the position estimate to be within the confines of the road.

Note now that if $\mathbf{Q} \rightarrow \infty$-i.e., as the uncertainty in the mobility goes to infinity-then (23) gives $\overline{\boldsymbol{P}}=\mathbf{J}_{\mathbf{P}}$. Therefore, it is clear how including the mobility in localization is important for the improvement of position estimates. In general, for a linear system with a Gaussian-noise distribution, the Kalman filter can be shown to achieve this CRB [16]. In our case, we are deploying an EKF where we are linearizing the nonlinear measurement equation using the Taylor series. Although, generally speaking, the EKF will not achieve the CRB, it still serves as a good ultimate bound. Generally, how close the EKF comes to the bound is based on how well the Taylor-series expansion describes the nonlinear measurement equation.

To see how well our algorithm performs in relation to the bound, we will first consider when there is no hard constraints imposed to force position estimates to be within the confines of the road. Therefore, we can use (23) to compare to the output of our algorithm. Consider the case where we use a ToA-based-radio-ranging scheme with a standard deviation of $4 \mathrm{~m}$ of error in the intervehicle-distance estimates. From Fig. 6, it is clear that due to the Taylor-series approximation, our algorithm without hard lane constraints (the top curve) does not always achieve the CRB (the middle curve). However, when hard lane constraints are imposed, the bound (23) can be exceeded (as seen by the lower curve). This result makes sense since, by imposing hard lane constraints, there is a new piece of information, therefore allowing our approach to exceed the $\mathrm{CRB}$ of the general velocity case. Indeed, the true CRB should be rederived for the case with lane constraints.

\section{B. Computational Cost}

In this section, we find the computational cost of our algorithm. Let $n$ be the number of vehicles within the local cluster. The computational complexity of our algorithm is $\mathcal{O}\left(m^{2.4}+\right.$ $n^{2}$ ) [22], where $m$ is the size of our measurement vector. The $m^{2.4}$ term is due to the multiplication of the $\hat{\mathbf{H}}_{k}$ matrix, and the $n^{2}$ term is for other matrix multiplications in the Kalman update. If all measurements are used, then $m$ will be equal to $\left(n^{2}-n\right)$; however, in practice, the distance measured between vehicles $i$ and $j$ are averaged so that $m$ is effectively equal to $\left(n^{2}-n\right) / 2$. However, for the majority of practical scenarios, $m$ is small. As a point of reference, our algorithm implemented in MATLAB runs in less than $500 \mathrm{~ms}$ on a $3-\mathrm{GHz}$ machine when there are up to 15 vehicles in a cluster and when the measurements are being shared at a rate of once per second.

The only stage in the algorithm that entails communication overhead is the step where the vehicles share distance and velocity estimates with their neighbors. If we assume that nonoverlapping clusters do not share the same channel because of range limitations, then the communication overhead is $\mathcal{O}\left(n^{2}\right)$ since $n^{2}$ measurements are being shared. In practice, this is implemented by each vehicle sending one packet of size $\mathcal{O}(n)$ containing all the observed distance measurements and a packet of constant size for the vehicle's velocity information.

Based on the frequency the measurements are shared between vehicles and the cluster size, the communication overhead becomes an interesting problem since there will be message collisions. For our experiments, we have used a 1-s update rate with cluster sizes not larger than 15 vehicles, but different update rates will be studied in the future.

\section{Simulation Results}

We study the performance of our algorithm under three scenarios. First, we compare our algorithm to the robust quads proposed by Moore et al. [14]. The robust-quads algorithm is designed primarily for localizing a stationary set of nodes and, therefore, does not have a mechanism for incorporating past localization results. To compare the robust-quads algorithm to ours, we operate it in a sequential fashion such that at each time step, the localization algorithm is run independent of the previous localization results. Next, we will compare our algorithm to the one presented in [23], where velocity and road boundary information is used to localize vehicles with a nonlinear least squares (NL-LS) optimization. Therefore, unlike the robust-quads implementation, past vehicle locations are incorporated into the estimation of the position at the current time step.

In the next two sections, we will outline the simulation environment used as well as define the performance metrics that we will be using to analyze and compare our localization algorithm to previously proposed ones.

\section{A. Simulation Environment}

We have used the Corridor Simulator, which is a microscopic transportation simulator and has been developed by the U.S. Federal Highway Administration to model vehicle movements. We have modeled a 4-km road with three east-bound and three west-bound lanes with vehicles entering into the east and west ends of the system at a rate of 1200 vehicles per hour, as shown in Fig. 7. The speed limit for the road was set to $100 \mathrm{~km} / \mathrm{h}$. 


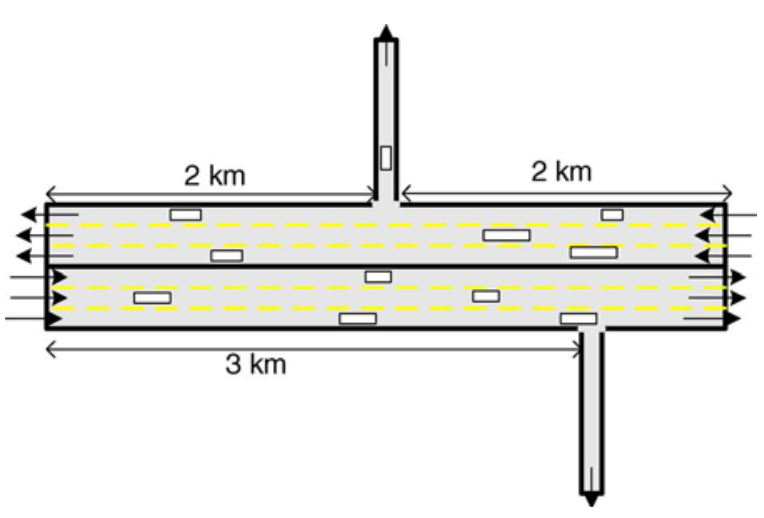

Fig. 7. Roadway for simulated vehicular environment.

For the experiments, we track a single vehicle's ability to determine the relative position of all vehicles within its cluster (i.e., all vehicles within its communication range of $150 \mathrm{~m}$ ) as it traverses the 4-km section of the road. Naturally, there will be a variable number of vehicles within a vehicle's communication range as it transverses the network. Given this experiment setup, we found that each vehicle generally had between 8 and 14 neighboring vehicles at each time step.

In addition, note that, for the simulation experiments, we have assumed that the position estimate for a vehicle when it first enters the cluster has been made via the GPS for the algorithms requiring an initial position estimate of the vehicle. After a vehicle has appeared once in the cluster, all future position estimates are based on prior estimates. The GPS position estimate for these experiments was set to differ from the true position by a Gaussian-distributed random variable with a standard deviation of $6 \mathrm{~m}$ that is for consistency with the real GPS error levels of 3-10 m [24].

\section{B. Performance Metrics}

There are two main metrics that we have chosen to use for evaluating the effectiveness of the localization algorithms. The first metric that we have used is the root-mean-square error (RMSE) in the final position estimate, which can be thought of as the average distance of the final position estimate from the actual position. We have defined RMSE in the final position estimate as follows:

$$
\begin{aligned}
& \sigma_{\text {final }} \\
& =\sqrt{\sum_{i=1}^{n} \frac{\left(x_{\text {final est. } i}-x_{\text {actual } i}\right)^{2}+\left(y_{\text {final est. } i}-y_{\text {actual } i}\right)^{2}}{n}}
\end{aligned}
$$

where $n$ is the number of vehicles in the cluster at the current time, $\left(x_{\text {actual } i}, y_{\text {actual } i}\right)$ represents the actual position of vehicle $i$ (often referred to as the ground truth in other literature), and $\left(x_{\text {final est. } i}, y_{\text {final est. } i}\right)$ is the position estimate of vehicle $i$ after running step 4 ) of our algorithm. Note that since only one vehicle is fixed at position $(0,0)$ when the position estimate is being made, an arbitrary reflection or rotation from the actual positions of the vehicles is an equally valid solution.

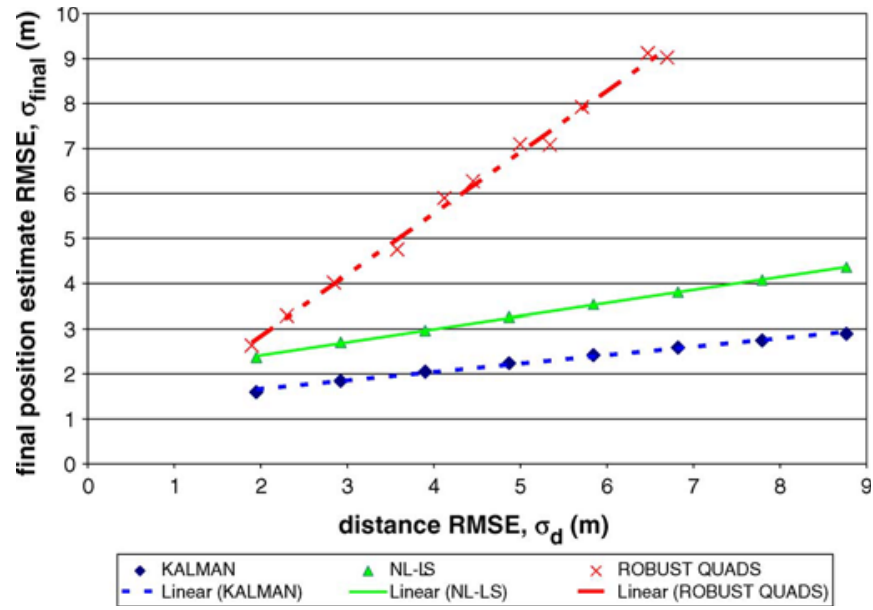

Fig. 8. Showing effect on performance when the intervehicle-distanceestimation error is varied.

The second metric that we have used is the RMSE in the intervehicle-distance measurements, which we have defined as

$$
\sigma_{d}=\sqrt{\sum_{i, j=1}^{M} \frac{\left(\hat{d_{i, j}}-d_{i, j}\right)^{2}}{M}}
$$

where $M$ is the number of intervehicle-distance measurements, $\hat{d_{i, j}}$ is the measured distance between vehicles $i$ and $j$, and $d_{i, j}$ is the actual distance between vehicles $i$ and $j$. This metric is important because it allows us to measure the sensitivity of the localization algorithm to error levels in the distance measurements.

\section{Comparisons With Other Algorithms}

First, we will compare the performance of our algorithm with the robust-quads algorithm [14]. Since the robust-quads algorithm does not rely on the initial position estimates of the vehicle, we will compare it to ours based on changing the error levels in the intervehicle-distance measurements.

To examine the effect of errors in the intervehicle-distance estimates on the final position estimate, we have varied the RMSE in the distance measurements while leaving all other parameters constant. The results of this experiment are shown in Fig. 8, where each data point is the average of ten runs of the algorithms through our simulated vehicular environments. The top curve shows the robust-quads algorithm, whereas the bottom curve is our proposed algorithm. From the figure, it is clear that the error level in position estimates of the robustquads algorithm grows at a linear rate which is about seven times greater than our algorithm. The major difference in performance can be attributed to the fact that the robust-quads algorithm does not make use of velocity information or road constraints.

To verify this claim and to see the benefits of the road and velocity information, we will compare our algorithm to the NLLS approach presented in [23]. Comparing the middle curve in Fig. 8, which is the results of the algorithm presented in [23], with the upper curve showing the robust-quads algorithm [14], 


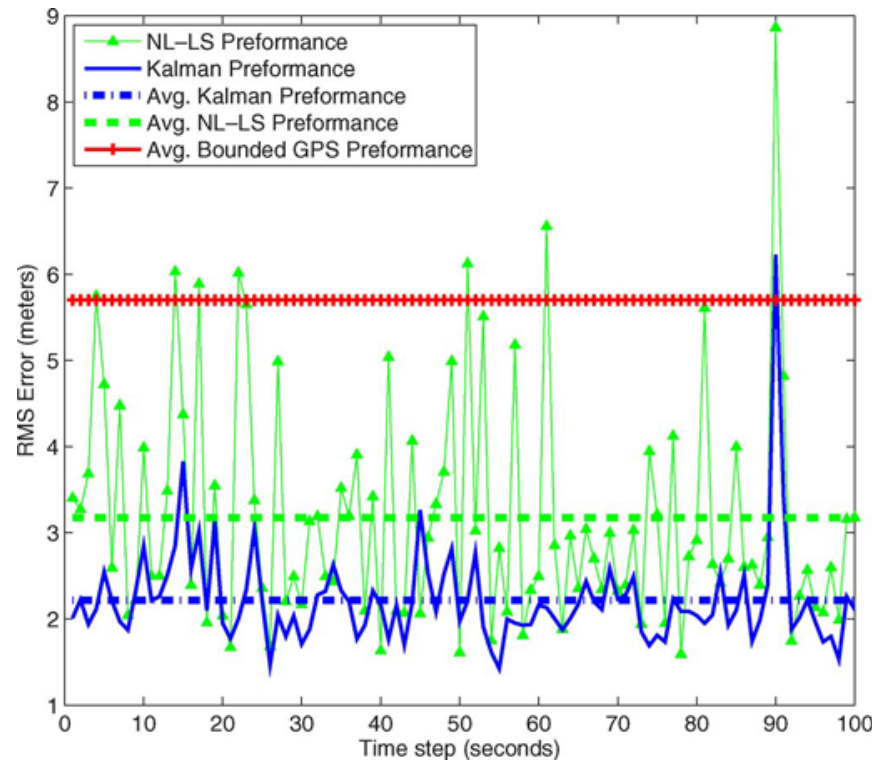

Fig. 9. Comparison of NL-LS approach to our Kalman-filter-based approach. The performance of using GPS with a mapping module is also shown for reference.

it is clear that by including velocity and road constraints, the sensitivity to distance-measurement errors is reduced. Although the algorithm presented in [23] shows significant performance gains over existing static-based localization schemes by including these additional constraints, on average, it performs worse than the algorithm proposed here. The primary reason for this performance difference is in how the velocity and road constraints are incorporated into the optimization scheme, as discussed earlier. This can be verified by examining the result of how well a single vehicle was able to localize its neighbors at each time step as it traveled through the system. If we assume that a Gaussian random variable with a standard deviation of $6 \mathrm{~m}$ describes the noise characteristics of the intervehicledistance measurements, then we get the results shown in Fig. 9. The curve with the higher peaks is the NL-LS implementation proposed in [23], and the bottom curve is our Kalman-filterbased approach presented in this paper. Overall, we see that there is much less variation in the error levels of our algorithm versus the NL-LS approach. This lower variation results in an overall lower average level of error for our algorithm-2.2 m for the Kalman-filter approach versus $3.2 \mathrm{~m}$ for the NL-LS approach.

In Fig. 9, we have also included the average performance of a GPS-based system (i.e., the horizontal line with the rms error of approximately $5.7 \mathrm{~m}$ ), which made use of lane and velocity constraints to establish position estimates. The GPS system was set to have an average accuracy of $6 \mathrm{~m}$, which is consistent with the performance of real-world GPS [24], and then, the velocity lane constraints were imposed. This reinforces the additional benefits of using intervehicle-distance estimates.

\section{CONCLUSION}

The pursuit of cooperative-vehicle-collision-warning system is one of the many efforts of automotive manufactures, gov- ernment, and researchers to reduce the vehicle-accident rate. In the future, it is envisioned that vehicles will be equipped with DSRC devices, which allows vehicle-to-vehicle and vehicle-toinfrastructure communications. Equipping vehicles with DSRC devices will allow vehicles to cooperatively share information (i.e., location, speed, heading, acceleration, etc.) with their peers and be able to predict potential hazards that lie on the road ahead. However, the ultimate success of this system will be dependent on how accurately the relative position of neighboring vehicles can be predicted. This is an essential component for cooperative-collision-warning systems.

The GPS and some of its improved variants are currently used for positioning; however, these technologies do not support the stringent reliability and accuracy requirements for cooperative-vehicle-collision-warning systems. In the effort to improve reliability and accuracy, radio-based-ranging techniques can be used to determine intervehicle distances. The RSSI, with an accurate model of the wireless channel, and the two-way reciprocal ToA system are two candidates.

In this paper, we discussed that a majority of the existing localization approaches are not applicable to vehicle networks for one or more of the following reasons: They do not account for the noise that is present in the internode-distance measurements, the node localization cannot be performed in a distributed fashion, or they fail with node mobility. Nevertheless, the robust-quads algorithm seemed to show promise for use in vehicular networks. However, the algorithm is still unproven for networks with high levels of node mobility.

It is likely that not one technology or one positioning algorithm, on its own, will provide the optimal solution for vehicle positioning within a cluster. However, using other resources (e.g., a vehicle's velocity and acceleration, a road map of the surrounding area, and the intervehicle distances) may improve the estimation of vehicle position and ultimately allow the realization of a cooperative-collision-warning system.

We have shown that gains in accuracy and reliability can be achieved over the GPS-based approaches by using intervehicledistance measurements taken by a radio-based-ranging technique and that the accuracy of localization can be improved by taking into account extra information, such as maps of the road, vehicle kinematics, etc. We have performed an analysis of our algorithm to examine its performance limits, computational complexity, and its sensitivity-measurement errors. We have shown that our Kalman-filter-based solution is accurate and reliable, and it allows a real-time implementation. The structure of our algorithm allows us to place a probabilistic confidence on the position estimates, so that inaccurate position estimates can be actively identified, making it practical for future vehicular safety applications.

\section{REFERENCES}

[1] J. Zhu and S. Roy, "MAC for dedicated short range communications in intelligent transport system," IEEE Commun. Mag., vol. 41, no. 12, pp. 60-67, Dec. 2003.

[2] National Highway Traffic Safety Administration, Report and press release the economic impact of motor vehicle crashes, 2000.

[3] R. Tatchikou, S. Biswas, and F. Dion, "Cooperative vehicle collision avoidance using inter-vehicle packet forwarding," in Proc. IEEE GLOBECOM Conf., Nov. 2005, pp. 2762-2766. 
[4] V. Kukshya, H. Krishnan, and C. Kellum, "Design of a system solution for relative positioning of vehicles using vehicle-to-vehicle radio communications during GPS outages," in Proc. IEEE VTC, Sep. 2005, pp. 1313-1317.

[5] R. Miller and Q. Huang, "An adaptive peer-to-peer collision warning system," in Proc. IEEE VTC, 2002, vol. 1, pp. 317-321.

[6] M. Grewal and L. Weill, Global Positioning Systems, Inertial Navigation, and Integration. Hoboken, NJ: Wiley, 2001.

[7] G. M. Djuknic and R. E. Richton, "Geolocation and assisted GPS," Computer, vol. 34, no. 2, pp. 123-125, Feb. 2001.

[8] S. Saha, K. Chaudhuri, D. Sanghi, and P. Bhagwat, "Location determination of a mobile device using IEEE 802.11b access point signals," in Proc. IEEE WCNC, Mar. 2003, pp. 1987-1992.

[9] A. Savvides, M. Srivastava, S. Basagni, M. Conti, S. Giordano, and I. Stojmenovic, Eds., Mobile Ad Hoc Networking. New York: IEEE Press, Dec. 2003, pp. 233-234.

[10] D. D. McCrady, L. Doyle, H. Forstrom, T. Dempsey, and M. Martorana, "Mobile ranging using low accuracy clocks," IEEE Trans. Microw. Theory Tech., vol. 48, no. 6, pp. 951-958, Jun. 2000.

[11] S. Capkun, M. Hamdi, and J. Hubaux, "GPS-free positioning in mobile ad-hoc networks," in Proc. Hawaii Int. Conf. Syst. Sci., Jan. 2001, pp. 9008-9017.

[12] R. Iyengar and B. Sikdar, "Scalable and distributed GPS free positioning for sensor networks," in Proc. IEEE Int. Conf. Commun., May 2003, pp. 338-342.

[13] T. Eren, D. Goldenberg, W. Whiteley, Y. R. Yang, A. S. Morse, B. D. O. Anderson, and P. N. Belhumeur, "Rigidity, computation and randomization of network localization," in Proc. IEEE INFOCOM, Mar. 2004, pp. 2673-2684.

[14] D. Moore, J. Leonard, D. Rus, and S. Teller, "Robust distributed network localization with noisy range measurement," in Proc. 2nd ACM Conf. SenSys, Nov. 2004, pp. 50-61.

[15] R. Parker and S. Valaee, "Cooperative vehicle position estimation," in Proc. ICC, 2007, pp. 5837-5842.

[16] H. L. V. Trees, Detection, Estimation, and Modulation Theory: Part I. New York: Wiley, 1968.

[17] D. Simon, Optimal State Estimation, 1st ed. New York: WileyInterscience, 2006.

[18] P. Gill, W. Murray, and M. Wright, Practical Optimization. New York: Academic, 1981, pp. 96-98. 133-141.

[19] D. Simon and T. Chia, "Kalman filtering with state equality constraints," IEEE Trans. Aerosp. Electron. Syst., vol. 38, no. 1, pp. 128-136, Jan. 2002.

[20] F. Gustafsson and F. Gunnarsson, "Mobile positioning using wireless networks: Possibilities and fundamental limitations based on available wireless network measurements," IEEE Signal Process. Mag., vol. 22, no. 4, pp. 41-53, Jul. 2005.
[21] N. Patwari, J. N. Ash, A. O. Hero, R. L. Moses, and N. S. Correal, "Locating the nodes: Cooperative localization in wireless sensor networks," IEEE Signal Process. Mag., vol. 22, no. 4, pp. 54-69, Jul. 2005.

[22] S. Thrun, W. Burgard, and D. Fox, Probabilistic Robotics. Cambridge, MA: MIT Press, 2005.

[23] R. Parker and S. Valaee, "Vehicle localization in vehicular networks," in Proc. IEEE Veh. Technol. Conf., Sep. 2006, pp. 1-5.

[24] C. Drane and C. Rizos, Positioning Systems in Intelligent Transportation Systems. Boston, MA: Artech House, 1997, pp. 230-231.

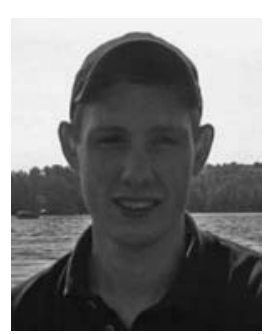

Ryan Parker received the B.Sc. degree in computer engineering from Queen's University, Kingston, ON, Canada, in 2005 and the M.Sc. degree in computer engineering from the University of Toronto, Toronto, ON, Canada, in 2006, where he studied wireless ad hoc vehicle networks.

From 2005 to 2006, he was a member of the Communications Group, University of Toronto. After finishing the M.Sc. degree at the end of 2006, he joined the Windows Mobile Division, Microsoft, Redmond, WA, as a Program Manager.

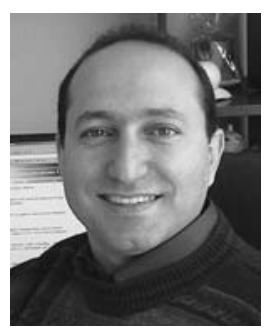

Shahrokh Valaee (S'88-M'89-SM'02) received the B.Sc. and M.Sc. degrees in electrical engineering from Tehran University, Tehran, Iran, and the Ph.D. degree in electrical engineering from McGill University, Montreal, QC, Canada.

From 1996 to 2001, he was an Assistant Professor with the Department of Electrical Engineering, Tarbiat Modares University, Tehran, and was with the Department of Electrical Engineering, Sharif University of Technology, Tehran. Since September 2001, he has been an Associate Professor with the Edward S. Rogers Sr. Department of Electrical and Computer Engineering, University of Toronto, Toronto, ON, Canada, where he is the Nortel Institute Junior Chair of Communication Networks.

Dr. Valaee is the Cochair for the Wireless Communications Symposium of the IEEE Global Telecommunications Conference 2006, the Editor for the IEEE Wireless Communications Magazine Special Issue on Toward Seamless Internetworking of Wireless LAN and Cellular Networks, and the Editor of a Special Issue of the Wiley Journal on Wireless Communications and Mobile Computing on Radio Link and Transport Protocol Engineering for Future Generation Wireless Mobile Data Networks. 